\title{
Kontrolliertes Trinken als Ziel und als Weg. Zwei Fallvignetten
}

\author{
Joachim Körkel $^{1}$ \\ Wilfried Langguth ${ }^{2}$ \\ Birgit Schellberg ${ }^{2}$ \\ Birgit $\mathrm{Neu}^{2}$
}

\author{
Controlled Drinking as Goal and as Means. Two Case Examples
}

\section{Zusammenfassung}

Es werden anamnestische Daten und der Entwicklungsprozess von zwei bisher durch die Suchthilfe nicht erreichten alkoholabhängigen Teilnehmern des „Ambulanten Gruppenprogramms zum kontrollierten Trinken (AkT)“ berichtet. Teilnehmer 1 (60 Jahre) zeigt auch noch 2 Jahre nach AkT-Ende eine weitgehend stabile Umsetzung des kontrollierten Trinkens mit deutlicher Trinkmengenreduktion (AkT-Beginn: $740 \mathrm{~g}$ Alkohol/Woche; AkTEnde: $260 \mathrm{~g}$; 2-Jahres-Katamnese: $300 \mathrm{~g}$ ) und damit einhergehender Zufriedenheit in zuvor alkoholbelasteten Bereichen (Partnerschaft, Arbeit). Teilnehmer 2 (56 Jahre) konnte seinen Wochenalkoholkonsum ebenfalls erheblich reduzieren (um 71\% zu AkT-Ende, um 44\% in der Halbjahreskatamnese), aber vor allem die alkoholfreien Tage nicht aufrechterhalten und alkoholbedingte Partnerschaftskonflikte nicht abwenden. Einzel- und Paargespräche führten 8 Monate nach AkT zu einer stationären Entgiftungsbehandlung und bis heute (2-Jahres-Katamnese) zur zufriedenen Abstinenz. Beide Fälle charakterisieren typische AkT-Verläufe. Sie zeigen, dass durch Angebote zum kontrollierten Trinken mehr Personen von der Suchthilfe erreicht werden können als bislang und kontrolliertes Trinken gelingen oder aber ein hilfreicher Zwischenschritt zur Abstinenz sein kann.

\section{Schlluisselwörter}

Kontrolliertes Trinken · Abstinenz · Suchthilfesystem

\section{Abstract}

Two case examples of alcohol-dependent Ss not treated for alcoholism so far and undergoing a German Behavioral Self-Control Training (group treatment; in German „Ambulantes Gruppenprogramm zum kontrollierten Trinken", AkT) are reported. Case 1 (age 60 years) can be rated as successful controlled drinker: he achieved a reduction in weekly alcohol consumption from $740 \mathrm{~g}$ (pre-assessment) to $260 \mathrm{~g}$ (end-of-treatment) and stabilised two years after treatment at $300 \mathrm{~g}$ with no more alcohol related problems in his partnership and at work. Case 2 (age 56 years) reduced his alcohol intake enormously, too ( $71 \%$ at endof-treatment, $44 \%$ half a year later) but he could not maintain his abstinent days and got increasing trouble with his wife. After several counselling sessions he decided for inpatient detoxification (8 months after AkT) and lives quite happily abstinent till today ( 2 years after AkT). Both cases exemplify typical courses of recovery with AkT: AkT leads people to seek treatment who were not inclined to undergo traditional abstinence-oriented treatment, and after AkT controlled drinking may be a realistic goal for them or an intermediate step to reach abstinence.

Key words

Controlled Drinking $\cdot$ Abstinence $\cdot$ Addiction Treatment System
Im Folgenden berichten wir anhand zweier Fallbeispiele die häufigsten Verläufe von Trinkmengenentwicklungen und Zielanpassungen im Rahmen des „Ambulanten Gruppenprogramms zum kontrollierten Trinken (AkT)“ (vergleiche zur allgemeinen Charakterisierung des AkT den Beitrag von Körkel et al., in diesem Heft).

${ }^{1}$ Evangelische Fachhochschule Nürnberg, Fachbereich Sozialwesen

2 Psychosoziale Beratungs- und Behandlungsstelle für Suchtkranke des Caritasverbandes Nürnberg 
Hans B. wendet sich auf Drängen seiner Lebenspartnerin, die über einen Zeitungsartikel auf das AkT gestoßen ist, an uns, d.h. die Psychosoziale Beratungs- und Behandlungsstelle für Suchtkranke des Caritasverbandes Nürnberg. Hans B. ist 60 Jahre alt und hat drei außer Haus lebende erwachsene Kinder, die der Ehe mit seiner vor längerer Zeit verstorbenen Frau entstammen und mit denen er weiterhin in regelmäßigem Kontakt steht. Er ist inzwischen wieder mit einer Lebenspartnerin liiert, die ihre eigene Wohnung bewohnt. Beruflich ist er als Hausmeister für eine größere Wohnanlage zuständig.

In den beiden dem AkT vorgeschalteten diagnostischen Gesprächen äußert Herr B. ausschließliches Interesse an einem Programm zum reduzierten Alkoholkonsum (wie dem AkT). Rein abstinenzorientierte Behandlungsangebote - über die als Alternativen zum kontrollierten Trinken gesprochen wird - lehnt er ab. Suchtbezogene Hilfe hat er bis zu diesem Zeitpunkt nicht beansprucht. Er erhofft sich durch das AkT eine deutliche Reduktion seines Alkoholkonsums, um weitere, auf sein Trinkverhalten zurückzuführende Spannungen innerhalb seiner Beziehung zu vermeiden (z.B. Vorwürfe und heftiger Streit wegen alkoholbedingter Ausfälligkeiten, Trennungsandrohungen usw.). Außerdem bereitet ihm seit geraumer Zeit das besonders im Rahmen seiner Hausmeistertätigkeit spürbare Nachlassen seiner Leistungsfähigkeit (früh einsetzende Abgespanntheit und häufige Unkonzentriertheit), das er ebenfalls mit seinem hohen Alkoholkonsum in Verbindung bringt, immer mehr Sorgen.

In den diagnostischen Vorgesprächen beziffert er die Menge an wöchentlich konsumiertem Bier, Wein und Schnaps - allen drei Alkoholika spricht er zu - auf umgerechnet 740 Gramm Alkohol (= 37 Standardeinheiten [SE]; 1 SE =0,51 Bier oder 0,2 l Wein oder 3 Schnäpse). Er nimmt jeden Tag Alkohol zu sich, abstinente Tage kommen nicht vor. Gemäß der ICD-10 ist er als (eher leicht) alkoholabhängig einzustufen (3 ICD-10-Kriterien erfüllt), eine körperliche Entzugssymptomatik wird nicht berichtet. Drei der 4 CAGE-Items werden mit ,ja“ beantwortet (ab 2 Zustimmungen: Alkoholproblem wahrscheinlich), der AUDIT ergibt 12 Punkte (ab 8 Punkten: problematischer Alkoholkonsum wahrscheinlich). Alkoholbedingte Folgeerkrankungen somatischer, psychischer oder sozialer Art liegen nicht vor, wie die ärztliche Untersuchung, der EuropASI und andere diagnostische Verfahren (z.B. GABS) ergeben.

Herrn B. wird nach den diagnostischen Vorgesprächen eine $\mathrm{Zu}$ sage für die AkT-Teilnahme gegeben. Mangels eines freien Platzes in der ersten AkT-Gruppe (beginnend im Oktober 1999) wird er der Wartekontrollgruppe zugeteilt. Während der $3 \frac{1}{2}$-monatigen Wartezeit bis zum Beginn seiner AkT-Gruppe verringert sich sein wöchentlicher Alkoholkonsum nur geringfügig von 37 auf 35 SE.

Ab Anfang Februar 2000 nimmt er regelmäßig an den 12 wöchentlich stattfindenden AkT-Gruppenabenden teil (in dieser AkT-Gruppe erstreckte sich das AkT auf 12 Sitzungen, in allen folgenden auf 10 Sitzungen). In der Gruppe beteiligt sich Herr B. rege und thematisiert u.a. seine Schwierigkeit, Trinkaufforderungen bei seiner beruflichen Tätigkeit als Hausmeister abzulehnen. Als für ihn besonders riskant erweist sich hierbei das Hei- zungsablesen, bei dem er - jahrelanger Gewohnheit folgend von einigen Mietern bereits mit alkoholischen Getränken erwartet und zum Konsum aufgefordert wird („ein Schnäpschen in Ehren ...“). Auch nach diversen im AkT durchgeführten Ablehnungsrollenspielen und der Hinterfragung seiner „Ja-Sage-Tendenz“ sieht er in Trinkanimationen während seiner Arbeitstätigkeit auch am Ende der Gruppensitzungen das größte Gefährdungspotenzial für seine Trinkkontrolle. Insbesondere bei beharrlichen Trinkverführungen stelle sich bei ihm ein „ungutes Gefühl“ ein, sofern er bei seiner Ablehnung bleibe. Letztlich erweist sich seine Befürchtung, bei konsequenter Ablehnung von Trinkeinladungen sein Gegenüber bleibend verstimmen zu können, als Auslöser dafür, „klein beizugeben“ und doch mitzutrinken - wenn auch lange nicht so viel wie in früheren Zeiten.

Sein Ausgangskonsum von $35 \mathrm{SE} /$ Woche ist bis Ende des AkT auf $13 \mathrm{SE} /$ Woche gesunken (vgl. Abb. 1). Im Durchschnitt legt er 1-2 abstinente Tage pro Woche ein. Der GGT-Wert reduzierte sich von 44,8 (vor AkT) auf 15,0 (nach Beendigung des Programms).

In der 6-Monats-Katamnese ist eine Verringerung der abstinenten Tage zu verzeichnen, die wöchentliche Trinkmenge bleibt demgegenüber stabil (13 SE/Woche, 1 abstinenter Tag alle 2 Wochen). Auch der GGT-Wert zeigt sich mit 15,8 kaum verändert. Die Zurückweisung von Trinkangeboten im Kontext seiner Hausmeistertätigkeit ist im Halbjahr nach Abschluss des AkT weiterhin sein neuralgischer Punkt. So überschreitet er mehrmals in o.g. Situationen das von ihm gesetzte Tageslimit (von 2-3 SE), kompensiert diese Überschreitung allerdings durch verringerten Konsum in den folgenden Tagen, um sein Wochenlimit einzuhalten. Bei dieser Stabilisierung seines Wochenkonsums spielt die Unterstützung durch seine Lebensgefährtin eine bedeutsame Rolle (Ermutigung, das Wochenmaximum an SE nicht zu überschreiten; Unterstützung beim gemeinsamen Einlegen alkoholfreier Abende usw.).

Zum Zeitpunkt der 1-Jahres-Nacherhebung ist der wöchentliche Konsum leicht von 13 auf 15 SE angestiegen, der GGT-Wert hat sich auf 19,9 erhöht. Umgekehrt hat er die Anzahl der abstinenten Tage auf drei innerhalb von zwei Wochen erhöht, was sein Bemühen, den leicht erhöhten Alkoholkonsum mancher Trinktage zu kompensieren, zum Ausdruck bringt.

Etwa zum Zeitpunkt der 1-Jahres-Nachbefragung beginnt Herr B., die aus dem AkT hervorgegangene Selbsthilfegruppe, die sich einmal pro Monat trifft, zu besuchen. Dort bringt er erneut die

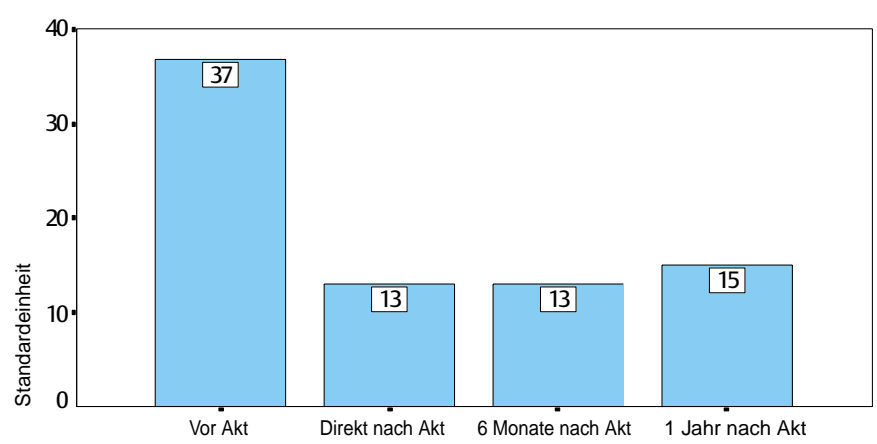

Abb. 1 Trinkmengenentwicklung bei Herrn B. (in Standardeinheiten pro Woche) 
Problematik der unzureichenden Ablehnung von Trinkaufforderungen im beruflichen Umfeld ein. Obwohl ihm dieses Problemfeld zeitweise zu schaffen macht, ist er insgesamt mit seinem Trinkverhalten zufrieden und sieht momentan weder Bedarf nach weitergehender Behandlung noch nach weiterer Trinkmengenreduzierung. Die vor dem AkT angespannte Paarsituation ist einem weitgehend harmonischen Miteinander gewichen - vor allem infolge des deutlich reduzierten Trinkverhaltens. Beruflich erlebt er sich wieder „fit“ und so gut wie nie durch seinen Alkoholkonsum gehandicapt - selbst wenn er Mietern bisweilen „auf ein Schnäpschen" einwilligt (es dann aber auch bei diesem einen belässt). Stolz macht ihn, dass er Tage, an denen er seine selbst gesetzte Konsumobergrenze (nämlich $2 \mathrm{SE}$, bei einem abstinenten Tag pro Woche überschreitet), durch vermehrte abstinente Tage zu kompensieren versteht und auf diese Weise sein Wochenlimit weitgehend einhält. Das wird dadurch begünstigt, dass er die Überschreitungen seines selbst gesetzten Tageslimits nicht als Drama mit damit einhergehenden Scham- und Schuldgefühlen verbucht, sondern konstruktiv als Anlass zum Ergreifen von Gegensteuerungsmaßnahmen zu nutzen versteht. Die Tatsache, dass sein Alkoholkonsum auch $1 \mathrm{Jahr}$ nach Ende des AkT um ca. $60 \%$ niedriger ist als in den davor liegenden Jahren, führt er auf ein durch das AkT geschaffenes Bewusstsein über die getrunkene Menge zurück. Er beschreibt dies als das Empfinden „so, jetzt langt's“, das nach 2-4 SE/Tag einsetzt. Mit anderen Worten hat er einen inneren Gradmesser für die getrunkene Alkoholmenge als selbststeuerungsrelevante Größe entwickelt.

Bei einer telefonischen Nachbefragung im März 2002, also 2 Jahre nach Beendigung des AkT, gibt Herr B. seine Trinkmenge mit weiterhin durchschnittlich 15 SE pro Woche an. Weiter berichtet er von einigen wenigen „Ausrutscher-Wochen“ mit 20-25 SE/ Woche, zu denen es zwischen dem ersten und zweiten Jahr nach AkT gekommen ist. Diese Erhöhungen traten jeweils in den bereits bekannten problematischen Hausmeistersituationen (z.B. nach dem Ablesen der Wasseruhren), aber auch bei zwei Festen (u. a. seiner Geburtstagsfeier) auf.

Um die Anzahl der abstinenten Tage wieder zu steigern und sich in Situationen mit bedrängenden Trinkanimationen achtsamer zu verhalten, hat er sich zum erneuten Führen des Trinktagebuchs, das er kurz nach Ende der AkT-Teilnahme aufgegeben hatte, entschlossen. Insgesamt ist er jedoch mit der erreichten Reduktion seiner Trinkmenge zufrieden. In der AkT-Selbsthilfegruppe fühlt er sich wohl und möchte diese weiterhin besuchen.

\section{Fall 2: Kontrolliertes Trinken - auch ein Weg zur Abstinenz}

Der 56-jährige Franz A. stellt sich in unserer Beratungsstelle mit dem Wunsch nach Teilnahme am AkT vor, auf das er durch einen Zeitungsbericht aufmerksam geworden ist. Suchtbezogene Hilfe hat er bislang noch nie gesucht. Herr A. lebt zusammen mit seiner Ehefrau in finanziell gesicherten Verhältnissen. Zu den zwei erwachsenen Kindern, die nicht mehr im Haus wohnen, besteht regelmäßiger Kontakt. Über die Familie hinaus unterhält er soziale Kontakte zu einer Reihe von Freunden und Verwandten. Beruflich ist er ganztägig als Software-Berater beschäftigt. Seinen Alkoholkonsum möchte er besser kontrollieren können, da es durch die nach höheren Alkoholmengen eintretende Unansprechbarkeit, zuweilen auch Reizbarkeit zu massiven verbalen und auch schon körperlichen Auseinandersetzungen mit seiner Ehefrau gekommen ist. Darüber hinaus machen ihm die seit etwa 2 Jahren aufgetretenen Konzentrationsstörungen beruflich sehr zu schaffen. Auf der anderen Seite wolle er den Alkohol nicht missen. Insbesondere unmittelbar nach Arbeitsende empfinde er die als Folge des rasch konsumierten Cognacs eintretende Entspannung und angenehme Schwere als sehr angenehm.

Nach Angabe in den beiden diagnostischen Einzelgesprächen vor dem AkT, in denen er ebenso wie in den ersten Gruppensitzungen gehemmt und unsicher wirkt, trinkt er bereits seit längerer Zeit relativ hohe Alkoholmengen: in der Regel ca. 1,5 I Bier und 0,41 Weinbrand an den Arbeitstagen (beginnend mittags, den Großteil am Abend), an den Wochenenden etwas weniger (im Wochendurchschnitt etwa 8,8 SE/Tag, 61,6 SE/Woche; keine abstinenten Tage). Nach der ICD-10-Diagnostik ergibt sich eine Alkoholabhängigkeit (4 ICD-10-Kriterien erfüllt). Im CAGE werden alle 4 Fragen mit ,ja“ beantwortet. Im AUDIT kommt er auf 25 Punkte. Daneben ist sein Nikotinkonsum beachtlich (15 Zigaretten/Tag). Körperliche Folgeerkrankungen des Alkoholkonsums sind bisher nicht aufgetreten, die Transaminasen liegen im Normbereich.

Im rechtlichen Bereich und anderen, per EuropASI abgeklärten potenziellen Problemfeldern sind keine Auffälligkeiten zu konstatieren.

Von Oktober bis Dezember 1999 (sowie einem weiteren Termin im Januar 2000) nimmt Herr A. sehr zuverlässig und engagiert an der ersten AkT-Gruppe teil. Schon nach den ersten 4 AkTAbenden gelingt ihm eine deutliche Konsumreduktion von anfangs täglich 8,8 SE auf 4,5 SE pro Trinktag. An den Wochenenden fällt ihm ein Übergang zur Abstinenz meist erstaunlich leicht. Trotz weiterer Reduktionsbemühungen und vielfältiger Anregungen dazu aus der Gruppe gelingt ihm keine weitere Trinkmengenreduktion während der Arbeitswoche. Auch Einsicht in die Funktionen seines Konsums (u. a. Stressbewältigung und Entspannung nach und zum Teil auch schon während der Arbeit) und die Erarbeitung von Strategien zum Umgang mit beruflichen Belastungen und zur Vermeidung von Risikosituationen (z.B. Nichtaufsuchen des Kiosks, an dem er seinen Cognac bezieht) verändern daran nichts. Von therapeutischer Seite wird ihm in einigen Sitzungen rückgemeldet, dass man erstaunt sei, mit welcher Energie er am kontrollierten Trinken festhalte, obwohl es ihm viel Kraft abverlange und letztlich nicht so gelinge, wie er es sich wünsche und er sich an den abstinenten Tagen offenbar sehr wohl fühle.

Am Ende des AkT liegt Herr A. bei einem Konsum von 4,5 SE/Tag und 3 abstinenten Tagen/Woche und somit bei einem Wochenkonsum von 18 SE (vgl. Abb. 2). Als Ziel äußert er, weiterhin kontrolliert trinken und die Trinkmenge zunehmend reduzieren $\mathrm{zu}$ wollen (auf 2-3 SE/Tag).

Sechs Monate nach Ende des AkT ist die tägliche Trinkmenge im Vergleich zum Programmende um 0,5 SE/Tag auf 5 SE/Tag leicht angestiegen. Im Wochenkonsum ergibt sich aber fast eine Verdoppelung der Menge auf $35 \mathrm{SE}$, da ihm keine abstinenten Tage mehr gelingen. Er ist nun - 6 Monate nach AkT-Ende - der festen Überzeugung, kontrolliertes Trinken ohne den regelmäßigen Rückhalt der AkT-Gruppe auf Dauer nicht mehr zu schaffen. 


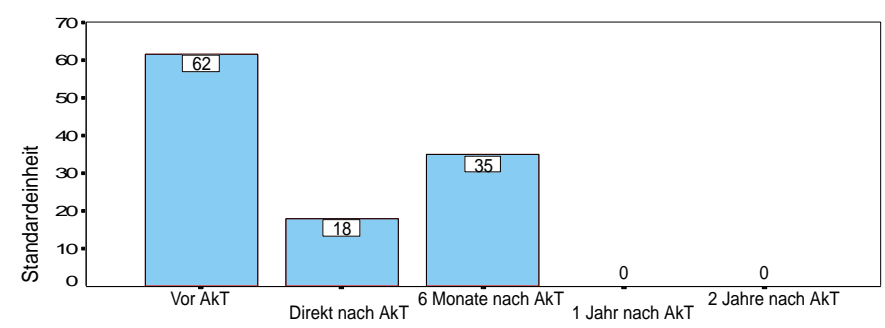

Abb. 2 Trinkmengenententwicklung bei Herrn A. (in Standardeinheiten pro Woche)

Die erneut aufgeflammten ehelichen Streitigkeiten angesichts seines wieder aufgenommenen Wochenendkonsums sowie der Wunsch, wieder alkoholfreie Arbeitstage einzulegen, bewegen ihn dazu, eine fortlaufende Einzelberatung in unserer Beratungsstelle wahrzunehmen.

Bereits zum ersten Beratungsgespräch kommt Herr A. mit der Vorstellung, zukünftig vielleicht besser „die Finger ganz vom Alkohol zu lassen". Zu dieser Überlegung sei er durch die positiven Erfahrungen, die er während des AkT mit seinen abstinenten Tagen gemacht habe und an denen er sich als wesentlich leistungsfähiger und aufgeschlossener erlebt habe, gelangt. Ganz sicher sei er sich allerdings nicht, ob er auf Dauer wirklich komplett ohne Alkohol auskommen wolle und könne. Aus diesem Grund wird im folgenden Beratungsgespräch vor dem Hintergrund seiner Erfahrungen aus dem AkT Bilanz der Vor- und Nachteile seines Alkoholkonsums bzw. der Abstinenz gezogen („4-Felder-Matrix“ [1]). Nach anschließendem Austausch mit seiner Ehefrau und einem weiteren zielabklärenden Beratungsgespräch entschließt er sich dann am Ende desselben zur dauerhaften Abstinenz. Um „einen radikalen Schnitt zu machen“, Abstand vom Alkohol zu gewinnen und die (leichten) körperlichen Entzugserscheinungen zu überwinden, entscheidet er sich sodann zu einer stationären Entgiftungsbehandlung in einem psychiatrischen Krankenhaus. Zum vierten und letzten Gespräch vor Antritt der Entgiftung wird die Ehefrau des Klienten hinzugezogen und die weiterhin vorhandenen gegenseitigen Vorwürfe und atmosphärischen Spannungen kommen zur Sprache. Beide sind nach diesem Gespräch „geschafft", haben aber die Zuversicht zurückgewonnen, dass ein von alten Hypotheken befreiter Neuanfang möglich sei.

Kurz nach diesem Paargespräch - 8 Monate nach Ende des AkT tritt Herr A. die stationäre Entgiftung an und kehrt nicht zuletzt durch die Wirkung, die sehr alkoholgeschädigte Entgiftungspatienten bei ihm hinterlassen haben, geläutert aus der Behandlung zurück. In den auf den Entzug folgenden 4 Monaten finden weitere sechs Beratungsgespräche statt, die sich schwerpunktmäßig mit der Wiederaufnahme alkoholfreier Freizeitaktivitäten und der Rückfallprophylaxe befassen (z. B. Vermeidung der permanenten beruflichen Überlastung). Herr A. bleibt über den gesamten Beratungszeitraum hinweg abstinent, was die eheliche Beziehung deutlich harmonisiert.

Auch zum Zeitpunkt der 1-Jahres-Katamnese im März 2001 ist Herr A. noch abstinent. Beim „AkT-Ehemaligenseminar“ (Ganztagestreffen der Teilnehmer aller bisher durchgeführten AkTGruppen) 2 Jahre nach Abschluss seiner AkT-Gruppe macht Herr A. einen zufriedenen Eindruck bei erhaltener Abstinenz. Er nimmt Anregungen auf, um auch zukünftig in Belastungssituationen seine Abstinenz zu bewahren. Weitere suchtspezifische Hilfe (z.B. Teilnahme an Selbsthilfegruppengesprächen) hat er in der Zwischenzeit nicht in Anspruch genommen und plant diese in naher Zukunft auch nicht ein.

\section{Diskussion}

Der Alkoholkonsum beider Männer hatte vor der AkT-Teilnahme erhebliche Ausmaße erreicht und war nach therapeutischer Einschätzung seit längerer Zeit dringend veränderungsbedürftig. Folgeerscheinungen sozialer und psychischer, zum Teil auch körperlicher Art waren bereits eingetreten. Trotzdem hatten beide Personen bislang keinerlei suchtspezifische Hilfe nachgesucht. Der berichtete Kontakt zur Psychosozialen Beratungs- und Behandlungsstelle für Suchtkranke war nur unter der Prämisse einer Reduktionsbehandlung zustande gekommen. Rein abstinenzorientierte Behandlungsangebote wurden abgelehnt. Bei beiden Personen ließ sich somit realisieren, was mit dem AkT und anderen Kontrollprogrammen wie dem „Ambulanten Einzelprogramm zum kontrollierten Trinken“ [2] zuallererst angestrebt wird: Personen mit Hilfebedarf an das Hilfesystem heranzuführen, um mit ihnen ein von Ideologien freies, zieladaptives Behandlungsprogramm durchzuführen.

Bei Herrn B. (Fall 1) erwies sich das AkT als hilfreich, um über den ganzen Beobachtungszeitraum von zwei Jahren nach dem AkT hinweg eine stabile und deutliche Reduzierung des Alkoholkonsums von letztlich etwa $60 \%$ zu erzielen - eine Größenordnung, die bei Kontrollprogrammen auch international des Öfteren berichtet wird (z. B. $[3,4])$. Wie bei vielen unserer AkT-Klienten, so wurde auch bei Herrn B. die Stabilisierung der Erfolge und die positive Gegenregulierung nach Ausrutschern durch unterstützendes Partnerverhalten erheblich begünstigt. Soziale Integration im Allgemeinen und Partnerunterstützung im Besonderen ist somit eine gute Voraussetzung für gelingende Veränderungsprozesse - auch beim kontrollierten Trinken [5]. Andererseits zeigt sich bei Herrn B., dass manche Problemfelder wie die fehlende Kompetenz, konsistent „nein“ bei Trinkaufforderungen zu sagen, weitergehender - ggf. psychotherapeutischer - Bearbeitung bedürften und nicht an 10 Gruppenabenden „abzuarbeiten“ sind.

Festzustellen bleibt schließlich, dass Herr B. seinen Konsum nicht gänzlich bzw. nicht immer auf das von der WHO bzw. der Britischen Ärztevereinigung für Männer empfohlene Niveau von maximal 30-40 g Alkohol/Tag (= $2 \mathrm{SE}$ ) bei 1-2 abstinenten Tagen pro Woche absenkte - was er allerdings auch nicht anstrebte. Bedenkt man seinen erheblichen Reduktionserfolg von $60 \%$ mit einhergehender gesundheitlicher Schadensminimierung und die Tatsache, dass er auf diesem Wege eine Normalisierung der vor der Behandlung beeinträchtigten Lebensbereiche (Partnerschaft, Arbeitsverhalten) erzielte und sich insgesamt mit den Veränderungen sehr zufrieden zeigt, kann seine Programmteilnahme als Erfolg gewertet werden. All dies schließt nicht aus, dass sich durch die zukünftige AkT-Selbsthilfegruppenteilnahme neue Impulse in Richtung weiterer Konsumreduktion respektive dauerhafter Abstinenz ergeben. 
Herr A. (Fall 2) konnte seinen Alkoholkonsum während des AkT um $71 \%$ senken und sich ein halbes Jahr nach AkT-Ende eine Reduktion von $44 \%$ gegenüber dem Ausgangsniveau bewahren. Trotz dieser auch nach einem Jahr noch beachtlichen Konsumminderung waren wesentliche Lebensbereiche (Partnerschaft, Arbeit) weiterhin durch den Alkoholkonsum tangiert. Diese Tatsache wie auch die während des Gruppenprogramms gesammelten positiven Erfahrungen mit alkoholfrei verbrachten Tagen begünstigten Herrn A.'s Interesse am Abstinenzziel und der Absolvierung einer Entgiftungsbehandlung als weiterem Schritt zu diesem Ziel. Die Mehrzahl unserer AkT-Teilnehmer, die sich letztlich für Abstinenz entschieden, haben an die Entgiftungsbehandlung eine stationäre Entwöhnungsbehandlung angeschlossen - bei Herrn A. erwies sich eine Entgiftungsbehandlung mit anschließenden Beratungsgesprächen als ausreichend. Herrn A.'s Fall demonstriert stellvertretend für viele andere, wie zwanglos und unspektakulär ein Übergang vom AkT zu abstinenzorientierten Hilfeangeboten und letztlich eine erfolgreiche Behandlung vonstatten gehen können, wenn keine Zieldogmatisierung erfolgt und auch in Kontrollprogrammen das Abstinenzziel als wertvolle Ergänzung bzw. Alternative zum Ziel des kontrollierten Trinkens im Gespräch gehalten wird. Wie von uns mehr- fach betont (z. B. [6]), erweisen sich Angebote zum kontrolliertes Trinken und zur Abstinenz somit nicht als Widerspruch, sondern als sinnvolle Ergänzung in einem ethisch legitimierten und auf die breite Masse problematischer Alkoholkonsumenten ausgerichteten Suchthilfesystem.

\section{Literatur}

${ }^{1}$ Miller WR, Rollnick S. Motivierende Gesprächsführung. Freiburg: Lambertus 1999

${ }^{2}$ GK Quest Akademie. Durchführung des „Einzelprogramms zum kontrollierten Trinken (EkT)“. (Seminarausschreibung) Heidelberg: GK Quest Akademie (info@GK-Quest.de) 2002

${ }^{3}$ Alden LE. Behavioral self-management controlled-drinking strategies in a context of secondary prevention. Journal of Consulting and Clinical Psychology 1988; 56: 280-286

${ }^{4}$ Miller WR, Leckman AL, Delaney HD, Tinkcom M. Long-term followup of behavioral self-control-training. Journal of Studies on Alcohol 1992; 53: 249-261

${ }^{5}$ Kruse G, Körkel J, Schmalz U. Alkoholismus erkennen und behandeln. Mit literarischen Beispielen. Bonn: Psychiatrie-Verlag 2001

${ }^{6}$ Körkel J. Kontrolliertes Trinken als neue Behandlungsoption. Neurotransmitter 2002; 13: 63-68 